\title{
Characteristics of the Innate Immune Response Associated with Cancer: Possible Therapeutic Targets
}

\author{
Amanda Pires Bonfanti*1,2 and Catarina Rapôso ${ }^{1}$ \\ ${ }^{1}$ Faculty of Pharmaceutical Sciences, State University of Campinas (UNICAMP), Brazil \\ ${ }^{2}$ Department of Structural and Functional Biology, Institute of Biology, (UNICAMP), Brazil
}

*Corresponding author: Amanda Pires Bonfanti, Faculty of Pharmaceutical Sciences, State University of Campinas (UNICAMP),

Campinas, São Paulo, Brazil, Email: amanda_bonfanti@hotmail.com

\section{ARTICLE INFO}

Received: 幽 April 09, 2020

Published: 慧 April 24, 2020

Citation: Amanda Pires Bonfanti, Catarina Rapôso. Characteristics of the Innate Immune Response Associated with Cancer: Possible Therapeutic Targets. Biomed J Sci \& Tech Res 27(2)-2020. BJSTR. MS.ID.004475.

Keywords: Immune Response; NK Cells; TAMs; Cancer

\section{ABSTRACT}

Immuno-oncology is an emerging field that is revolutionizing cancer treatment. Although most immunomodulatory strategies have focused on increasing the response of $\mathrm{T}$ cells, there has been a recent increase in interest in taking advantage of the natural killer cell (NK) compartment. The clinical responses to adoptive immunotherapy based on NK cells, however, were thwarted by the profound immunosuppression induced by the tumor microenvironment, particularly severe in the context of solid tumors. Tumorassociated macrophages (TAMs) have a prominent role in suppressing modulation of the tumor microenvironment, and these cells have been shown to be involved in inactivation of NK cells stimulated in vitro and transferred adoptively to cancer patients. The interaction between TAMs and NK cells can therefore be a prospective therapeutic target to improve cell therapies against cancer, and molecules that target these two cells have the potential to be immunoadjuvant agents.

Abbreviations: NK: Natural Killer; TAMs: Tumor-Associated Macrophages; CTLs: Cytotoxic T lymphocytes; ADCC: Antibody-Dependent Cell Cytotoxicity; IL: Interleukin; TNF- $\alpha$ : Tumor Necrosis Factor- $\alpha$; TGF: Tumor Growth Factor; VTGF: Vascular Tumor Growth Factor; FGF: Fibroblast Growth Factor

\section{Mini Review}

The development of cancer involves six categorical characteristics (cancer hallmarks):

1) Unregulated cell proliferation (due to self-sufficiency in growth signaling or insensitivity to inhibitory growth signals).

2) Evasion of programmed cell death.

3) Sustained angiogenesis.

4) Tissue invasion and metastasis.

5) Inflammation associated with the tumor and

6) Evasion of the immune response [1,2].

Pathologists have recognized that virtually all neoplastic lesions are infiltrated by cells from both the innate and adaptive immune responses, whose density varies from subtle infiltration to severe inflammation $[3,4]$. The immune system plays a role in combating the formation and progression of insipient neoplasms, late tumors and micro metastases, and clinical epidemiology gives evidence of this role of the immune response in some forms of human cancer $[5,6]$. For example, patients with ovarian and colon tumors that are heavily infiltrated with CD8+ cytotoxic T lymphocytes (CTLs) and natural killer cells (NK) have a better prognosis than those who lack these cells [4]. In mice, deficiencies in the function of CTLs, CD4+ T helper lymphocytes, or NK cells led to an increase in the incidence of neoplasms $[7,8]$.

According to this logic, in immunocompetent individuals, solid tumors have somehow managed to evade detection by the various aspects of the immune system [1]. Suppressor cells are found (predominantly) in the tumor microenvironment, such as regulatory T cells (Treg), a subtype of CD4+ T lymphocyte capable of suppressing immune responses [9], immature myeloid cells with suppressive capacity (MDSCs) [10] and tumor-associated macrophages (TAMs; which often have a suppressor profile - M2) [11]. In addition, in immunological interactions between the tumor 
and the host, highly immunogenic cancer cells can prevent immune destruction by disabling components of the immune system. For example, neoplastic cells can paralyze CTLs and infiltrating NK cells through the secretion of TGF- $\beta$ and other immunosuppressive factors (Yang, et al.) [12]. NK cells are innate effector cells that play a crucial role in inhibiting the development of cancer, due to their ability to recognize and lyse transformed cells without the need for prior sensitization [13]. This ability drew a lot of attention to NK cells as promising immunotherapeutic agents [11]. Its antitumor activity is regulated through a sophisticated network of inhibitory receptors and activators [14].

The first include Killer-cell immunoglobulin-like receptors (KIR), CD94 / NKG2A and ILT2 / LIR-1 / CD85j, all capable of making NK cells tolerant after binding to their own type I molecules of the histocompatibility complex (MHC I) $[15,16]$ The TGF- $\beta$ receptor also plays a role in suppressing cytokine-induced NK cell activation [17]; in addition, a relevant inhibitory receptor expressed by NK cells in cancer is programmed cell death protein 1 (PD-1) [18]. Activating receptors include natural NCRs cytotoxicity receptors (NKp30, NKp44, NKp46), type C lectin receptors [NKG2D (NK group 2, member D) and CD94 / NKG2C], the DNAM-1 adhesion / coactivation receptor (accessory DNAX-1 molecule) and the 2B4, CS1 (CD2 cell surface glycoprotein subset 1) and NTB-A (NK, T, $B$ antigen) $[15,16]$. These receptors interact with ligands highly expressed in the target cells after tumor transformation, viral infection and cell stress and trigger natural cytotoxicity of NK cells. In addition, NK cells express a CD16 receptor, which binds to the IgG Fc fragment and mediates antibody-dependent cell cytotoxicity (ADCC) [19].

However, to the detriment of their important antitumor role, the number and / or function of these lymphocytes is largely reduced during the progression of cancer. The correlation between the deficient function of NK cells and the development of metastases has been established in head and neck cancer $[20,21]$, pharyngeal [22] and other solid tumors $[23,24]$. NK cells in the peripheral blood of patients with cervical cancer have lower levels of expression of the activation receptors (NKp46, NKp30 and NKG2D) and the magnitude of this negative regulation is correlated with tumor progression [25]. Such observations stimulated the development of therapeutic approaches that aim to restore cytotoxicity mediated by intratumoral NK cells, blocking their inhibitory receptors or reducing the immunosuppressive factors present in the tumor microenvironment. NK cells do not attack healthy own tissues, nor do they induce a storm of inflammatory cytokines, enabling their use in allogeneic adoptive cell therapy [14,26,27].

Clinical responses to adoptive immunotherapy based on NK cells haven't been promising, due to the profound immunosuppression induced by the tumor microenvironment, being particularly severe in the context of solid tumors [11]. The tumor microenvironment makes NK cells dysfunctional, hindering cell proliferation, cytokine secretion, activator-receptor expressions and cytolytic activity [28-32]. As a result, adoptively transferred NK cells failed to demonstrate clinical benefits in solid tumors. For example, in a clinical trial, patients with metastatic melanoma or renal cell carcinoma showed no clinical response after the adoptive transfer of autologous NK cells activated in vitro, despite the fact that these cells efficiently lyse melanoma cells in culture [33]. This study demonstrated that, after the adoptive transfer, it was discovered that NK cells were quiescent and could no longer lyse tumor cells in vitro and expressed significantly lower levels of the main activating receptor (NKG2D).

These findings suggested that cells in the tumor microenvironment, other than tumor cells, have a role in inhibiting NK cell function, and evidence has shown that TAMs can play a crucial role in this suppression [11]. TAMs are found within the neoplastic tissue, as well as in the surrounding tissues [34] and can be pro- or anti-tumorigenic $[35,36]$. For, a suppressive profile predominates among TAMs [11]. Macrophages are classified by literature into two main groups, M1 and M2 (although this polarization is a simplistic concept and there is, in fact, a complex phenotypic spectrum). M1 macrophages are involved in the inflammatory response, pathogen clearance and antitumor immunity, through the expression of proinflammatory cytokines, such as interleukin (IL) -1 $\beta$, IL-6, IL-12, IL-23, tumor necrosis factor- $\alpha$ (TNF- $\alpha$ ) and inducible nitric oxide synthase (iNOS or $\mathrm{NOS}_{2}$ ) [34,37,38]. In contrast, M2 macrophages are known to promote tissue repair and remodeling, angiogenesis and promote tumor progression [39].

M2 macrophages release anti-inflammatory cytokines, such as IL-10 and TGF- $\beta$, in addition to tumor growth factor (TGF), vascular growth factor (VTGF), other pro-angiogenic factors, such as the basic fibroblast growth factor (FGF2), and enzymes that degrade the extracellular matrix, including matrix- 9 metalloproteinase (MMP-9) and other MMPs. Suppressive TAMs inhibit the Th1 antitumor response, while promoting the Th2 phenotype and Treg cell responses [40]. In addition, IL-10 secreted by TAMs inhibits the local production of IL-12, a crucial cytokine to trigger the cytotoxicity of NK cells $[41,42]$. A recent study demonstrated that both M2 macrophages (of peritoneal origin and generated from bone marrow), and TAMs, in coculture with NK cells, substantially inhibit the activation of the latter and their cytotoxicity against tumor cells. Both macrophages, M2 and TAMs, are producers of the immunosuppressive cytokine TGF- $\beta$ and the inhibition of this cytokine restored the cytotoxicity of NK cells in contact with the macrophages, implicating TGF- $\beta$ in this inhibition mechanism [11]. Therefore, reprogramming suppressive TAMs for a proinflammatory phenotype can change the course of the anti-tumor immune response.

Although it is known that M1 macrophages activate NK cells during the antineoplastic response [11] and that there is substantial evidence that TAMs have a prominent role in 
modulating the tumormicroenvironment, it is surprising that few studies have investigated the role of TAMs in NK cell dysfunction associated with neoplasms [11] and also, that the crosstalk between these two cells as an immunotherapeutic strategy has not been explored. The interaction between NK cells and TAMs can be a prospective therapeutic target to improve the efficacy of NK cells in cell therapies against cancer. For that reason, the manipulation of cells of the immune system, including macrophages and NK cells, have been tested as immunotherapy or even as adjuvants to other techniques in the treatment of cancer [43]. However, the difficulty of sustaining the activation of these cells in the tumor suppressor microenvironment is the main cause of clinical frustration; cellular immunotherapy combining macrophages and activated NK cells may be a way to overcome this obstacle.

Finally, it is relevant to mention that current treatment options for cancer, which often involve a combination of chemotherapy, radiation therapy and surgery, have been shown to be inefficient; in the case of glioblastomas, for example, the clinical course from the moment of diagnosis remains catastrophic and few patients achieve a 2.5-year post-diagnosis survival; the relative survival in the first year is $35 \%, 13.7 \%$ in the second year and less than $5 \%$ survive for 5 years after diagnosis $[44,45]$. Considering these limitations, efforts have been directed towards the development of new treatment strategies. In addition, another relevant point is that, while the usual chemotherapy against cancer causes early adverse events, compromising the defense mechanisms, the new classes of immune therapy through monoclonal antibodies and pro-inflammatory mediators, such as interleukins, can induce inflammatory responses. overwhelming and autoimmunity. Modulating the immune response through an agent that is not an immune mediator can generate a more specific response and with a lower risk of these side effects.

\section{Competing of Interests}

I declare that the authors have no competing of interests.

\section{Acknowledgements}

This work was supported by the following Brazilian foundations: Fundação de Amparo à Pesquisa do Estado de São Paulo (The São Paulo Research Foundation - FAPESP - grant number 2015/04194-0) and Conselho Nacional de Desenvolvimento Científico e Tecnológico (The Brazilian National Council for Scientific and Technological Development - CNPq - grant number 431465/2016-9). Amanda Pires Bonfanti is a fellow of FAPESP (\#2018/23559-7).

\section{References}

1. Hanahan D, Weinberg RA (2011) Hallmarks of cancer: the next generation. Cell 144(5): 646-674.

2. Anighoro A, Bajorath J, Rastelli G (2014) Polypharmacology: challenges and opportunities in drug discovery. J Med Chem 57(19): 7874-7887.
3. Dvorak HF (1986) Tumors: wounds that do not heal. Similarities between tumor stroma generation and wound healing. N Engl J Med 315(26): 1650-1659.

4. Pagès F, Galon J, Dieu Nosjean MC, Tartour E, Sautès Fridman C, et al. (2010) Immune infiltration in human tumors: a prognostic factor that should not be ignored. Oncogene 29(8): 1093-1102.

5. Bindea G, Mlecnik B, Fridman WH, Pagès F, Galon J (2010) Natural immunity to cancer in humans. CurrOpin Immunol 22(2): 215-222.

6. Ferrone C, Dranoff G (2010) Dual roles for immunity in gastrointestinal cancers. J Clin Oncol 28(26): 4045-4051.

7. Teng MWL, Swann JB, Koebel CM, Schreiber RD, Smyth MJ (2008) Immune-mediated dormancy: an equilibrium with cancer. J Leukoc Biol 84(4): 988-993.

8. Kim R, Emi M, Tanabe K (2007) Cancer immunoediting from immune surveillance to immune escape. Immunology 121(1): 1-14.

9. Frydrychowicz M, Boruczkowski M, Kolecka Bednarczyk A, Dworacki G (2017) The dual role of Treg in cancer. Scand J Immunol 86(6): 436-443.

10. Ostrand Rosenberg S, Sinha P (2009) Myeloid-derived suppressor cells: linking inflammation and cancer. J Immunol 182(8): 4499-4506.

11. Krneta T, Gillgrass A, Poznanski S, Chew M, Lee AJ, et al. (2017) M2polarized and tumor-associated macrophages alter NK cell phenotype and function in a contact- dependent manner. J Leukoc Biol 101(1): 285295.

12. Shields JD, Kourtis IC, Tomei AA, Roberts JM, Swartz MA (2010) Induction of lymphoid like stroma and immune escape by tumors that express the chemokine CCL21. Science 328(5979): 749-752.

13. Srivastava S, Lundqvist A, Childs RW (2008) Natural killer cell immunotherapy for cancer: a new hope. Cytotherapy 10(8): 775-783.

14. Fionda C, Stabile H, Molfetta R, Soriani A, Bernardini G, et al. (2018) Translating the anti-myeloma activity of Naural Killer cells into clinical application. Cancer Treat Rev 70: 255-264.

15. Gasser S, Orsulic E, Brown EJ, Raulet DH (2005) The DNA damage pathway regulates innate immune system ligands of the NKG2D receptor Nature 436(7054): 1186-1190.

16. Gilfillan S, Chan CJ, Cella M, Haynes NM, Rapaport AS, et al. (2008) DNAM1 promotes activation of cytotoxic lymphocytes by nonprofessional antigen-presenting cells and tumors. J Exp Med 205(13): 2965-2973.

17. Medicherla S, Li L, Ying Ma J, Kapoun AM, Gaspar NJ, et al. (2007) Antitumor activity of TGF-beta inhibitor is dependent on the microenvironment. Anticancer Res 27(6B): 4149-4157.

18. Benson DM, Bakan CE, Mishra A, Hofmeister CC, Efebera Y, et al. (2010) The PD-1/PD-L1 axis modulates the natural killer cell versus multiple myeloma effect: a therapeutic target for CT-011, a novel monoclonal anti-PD-1 antibody. Blood 116(13): 2286-2294.

19. Trinchieri G, Valiante N (1993) Receptors for the Fc fragment of IgG on natural killer cells. Nat Immun 12(4-5): 218-234.

20. Schantz SP, Campbell BH, Guillamondegui OM (1986) Pharyngeal carcinoma and natural killer cell activity. Am J Surg 152(4): 467-474.

21. Schantz SP, Ordonez NG (1991) Quantitation of natural killer cell function and risk of metastatic poorly differentiated head and neck cancer. Nat. Immun. Cell Growth Regul 10(5): 278-288.

22. Schantz SP, Savage HE, Racz T, Taylor DL, Sacks PG (1989) Natural killer cells and metastases from pharyngeal carcinoma. Am J Surg 158(4): 361- 366 .

23. Pross HF, Lotzova E (1993) Role of natural killer cells in cancer. Nat Immun 12(4-5): 279-292.

24. Imai K, Matsuyama S, Miyake S, Suga K, Nakachi K (2000) Natural cytotoxic activity of peripheralblood lymphocytes and cancer incidence: 
an 11-year follow-up study of a general population. Lancet 356(9244) 1795-1799

25. Garcia Iglesias T, Toro Arreola AD, Albarran Somoza B, Toro Arreola SD, Sanchez Hernandez PE, et al. (2009) Low NKp30, NKp46 and NKG2D expression and reduced cytotoxic activity on NK cells in cervical cancer and precursor lesions. BMC Cancer 9: 186.

26. Chiossone L, Dumas PY, Vienne M, Vivier E (2018) Natural killer cells and other innate lymphoid cells in cancer. Nat Rev Immunol 18(11): 671-688.

27. Sues WC, Lee WYW, Leung KT, Pan XH, Li G (2018) Natural Killer Cell based cancer immunotherapy: A review on 10 years completed clinical trials. Cancer Invest 36(8): 431-487.

28. Bauernhofer T, Kuss I, Henderson B, Baum AS, Whiteside TL (2003) Preferential apoptosis of CD56dim natural killer cell subset in patients with cancer. Eur J Immunol 33(1): 119-124.

29. Platonova S, Cherfils-Vicini J, Damotte D, Crozet V, Vieillard N, et al. (2011) Profound coordinated alterations of intratumoral NK cell phenotype and function in lung carcinoma. Cancer Res 71(16): 5412-5422.

30. Gati A, Rocha SD, Guerra N, Escudier B, Moretta A, et al. (2004) Analysis of the natural killer mediated immune response in metastatic renal cell carcinoma patients. Int J Cancer 109(3): 393-401.

31. Carrega P, Morandi B, Costa R, Frumento G, Forte G, et al. (2008) Natural killer cells infiltrating human nonsmall-cell lung cancer are enriched in CD56 bright CD162 cells and display an impaired capability to kill tumor cells. Cancer 112(4): 863-875.

32. Levy EM, Roberti MP, Mordoh J (2011) Natural killer cells in human cancer: from biological functions to clinical applications. J Biomed Biotechnol 2011: 676198.

33. Parkhurst MR, Riley JP, Dudley ME, Rosenberg SA (2011) Adoptive transfer of autologous natural killer cells leads to high levels of circulating natural killer cells but does not mediate tumor regression. Clin Cancer Res 17(19): 6287-6297.

34. Edin S, Wikberg ML, Dahlin AM, Rutegård J, Öberg A, et al. (2012) The distribution of macrophages with M1 or M2 phenotype in relation to prognosis and the molecular characteristics of colorectal cancer. PLoS One 7(10): e47045.

ISSN: 2574-1241

DOI: 10.26717/BJSTR.2020.27.004475

Amanda Pires Bonfanti. Biomed J Sci \& Tech Res

(C) This work is licensed under Creative

Submission Link: https://biomedres.us/submit-manuscript.php
35. Mantovani A, Bottazzi B, Colotta F, Sozzani S, Ruco L (1992) The origin and function of tumor-associated machophage. Immunol Today 13(7): 265-270.

36. Engstrom A, Erlandsson A, Delbro D, Wijkander J (2014) Conditioned media from macrophages of M1, but not M2 phenotype, inhibit the proliferation of colon cancer cell lines HT-29 and CACO-2. Int J Oncol 44(2): 385-392

37. Laoui D, Overmeire EV, Baetselier PD, Ginderachter JAV, Raes G (2014) Functional Relationship between tumor-associated macrophages and macrophage colony-stimulating factor as contributors to cancer progression. Front Immunol 5: 489.

38. Tran TH, Amiji NM (2015) Targeted delivery systems for biological therapies of inflammatory diseases. Expert Opin Drug Deliv 12(3): 393414.

39. Mantovani A, Schioppa T, Porta C, Allavena P, Sica A (2006) Role of tumor-associated macrophages in tumor progression and invasion. Cancer Metastasis Rev 25(3): 315-322.

40. Ostuni R, Kratochvill F, Murray PJ, Natoli G (2015) Macrophages and cancer: from mechanisms to therapeutic implications. Trends Immunol 36(4): 229-239.

41. Sitkovsky MV, Ohta A (2005) The "danger" sensors that STOP the immune response: the A2 adenosine receptors? Trends Immunol 26(6): 299-304.

42. DeNardo DG, Barreto JB, Andreu P, Vasquez L, Tawfik D, et al. (2009) CD4+ T cells regulate pulmonary metastasis of mammary carcinomas by enhancing protumor properties of macrophages. Cancer Cell 16(2): 91-102.

43. Da Silva IA, Chammas R, Lepique AP, Jancar S (2017) Plateletactivating factor (PAF) receptor as a promising target for cancer cell repopulation after radiotherapy. Oncogenesis 6(1): e296.

44. Ostrom QT, Gittleman, Farah P, Ondracek A, Chen Y, et al. (2013) CBTRUS statistical report: primary brain and central nervous system tumors diagnosed in the United States in 2006-2010. Neuro Oncol 2(Suppl 2): ii1-56.

45. Smoll NR, Schaller K, Gautschi OP (2013) Long-term survival of patients with glioblastoma multiforme (GBM). J clin Nuerosci 20(5): 670-675.

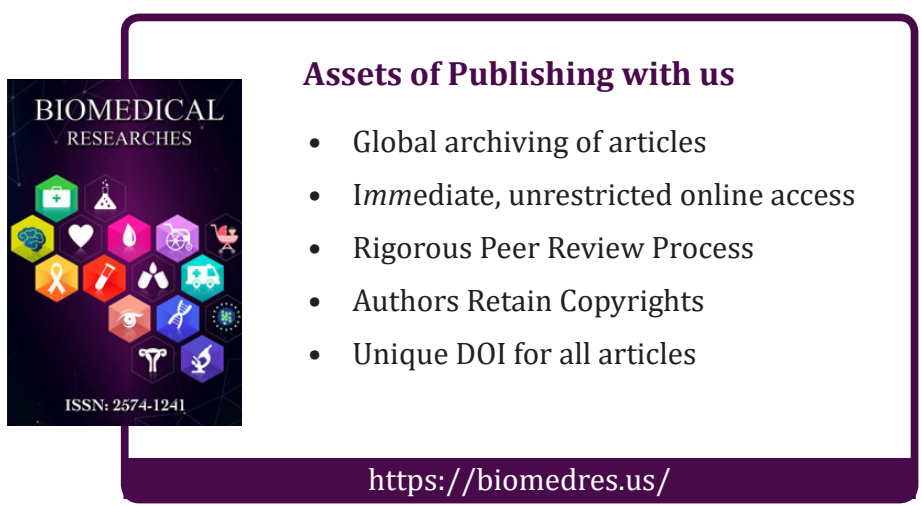

